
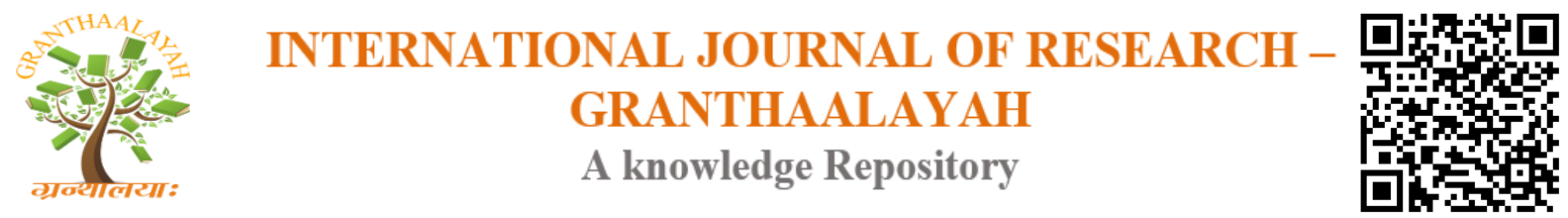

Science

\title{
PROFILE OF STUDENT ALGEBRA THINKING ABILITY BASED ON MATHEMATICAL PRELIMINARY SKILLS
}

\author{
Hestu Wilujeng *1 \\ ${ }^{* 1}$ University of Muhammadiyah Tangerang, Indonesia
}

\begin{abstract}
This study aims to explore and analyze the students' algebraic thinking skills that have early mathematical abilities. This research is an explorative research with qualitative approach. The Subject consists of 3 students who have high early, medium and low mathematical ability. The research instrument is self-researcher with supporting instrument that is about the algebra thinking ability test and interview guidance. The ability to think algebra includes problemsolving skills, mathematical modeling and generalization. Data analysis used qualitative descriptive analysis with guidance indicators of algebraic thinking ability while data analysis of interview uses data reduction step, data presentation, and decision making. Testing data validity using method triangulation. The result of the research is that students with high mathematical preliminary skills (MPS) can solve the problem well, MPS students are able to understand and plan the settlement well, but the mathematical modeling is not accurate so result in the settlement whereas the low MPS students in understanding the problem just read but do not have the solution plan.
\end{abstract}

Keywords: Profile; Algebraic Thinking; Mathematical Preliminary Skills.

Cite This Article: Hestu Wilujeng. (2017). "PROFILE OF STUDENT ALGEBRA THINKING ABILITY BASED ON MATHEMATICAL PRELIMINARY SKILLS.” International Journal of Research - Granthaalayah, 5(11), 210-216. 10.29121/granthaalayah.v5.i11.2017.2349.

\section{Introduction}

Algebra is one of the branches that is considered frightening in mathematical material in the School. A prospective teacher recounts his past experiences while learning algebra. Mathematical material can be well understood before learning algebra material in junior high school because it finds abstract symbolic language so it is not easy to understand its meaning (Radford, 2012). The transition from arithmetic to algebra is quite difficult for students as it requires students to make many adjustments even students who are quite capable in arithmetic. Kieran (2004) suggests an algebra learning adjustment that focuses on relationships rather than just counting numbers, focusing on representing and solving problems and focusing on the meaning of the same signs. Most students if given the problem of algebra they directly calculate 
without understanding the meaning of the question, the relationship of each sentence so that students difficulty in solving problems and represent the problem.

Based on preliminary research conducted by researchers about the ability of algebra readiness it is found that the ability of students is still lower that is less than 50\%. The students' difficulties in reading ability and declaring various forms of numeracy, calculating ability, determination of the largest federal factors and least common multiple, ability to use comparative concepts to illustrate problems and searching ability, surface area and volume. The low ability of students' algebra preparedness has an effect on algebraic thinking ability (Wilujeng, 2016).

Algebraic thinking ability by Kieran \& Chalouh (1993) is a thinking skill that involves the development of mathematical reasoning by building meaning of the symbols and algebraic operations. By thinking algebra students are accustomed to perform activities such as analyzing, generalizing, solving problems, forming patterns that are presented either in the form of tables, words, images, diagrams and mathematical expressions. Based on Kieran's (2004) opinion, algebraic thinking consists of generational activity, transformational activity and meta-global level activity. These activities involve students on generalizability, mathematical modeling, mathematical representation, problem solving and proving.

Algebraic thinking according to Lew (2004) is a thinking process that includes six mathematical thinking abilities consisting of generalizations, abstractions, analytical thinking, dynamic thinking, modeling and organization. Radford (2006) argues that the algebraic thinking characteristic of algebraic thinking begins with a person's sensitivity about something / objects that cannot be determined exactly then followed by an analysis of the object and the last is to model objects that have been analyzed in symbols. Based on the opinion of experts about algebraic thinking skills in this study algebra thinking ability that researched consists of mathematical modeling, problem solving and generalization. This is in accordance with the NCTM (2000) stating that at grade VIII junior level should have the ability to understand, analyze and generalize the form of the pattern with tables, graphs, words and rules of symbols; represent and analyze mathematical situations and use algebraic symbols; using mathematical modeling to solve algebraic problems.

Ability to think algebra in this research is problem solving and mathematical modeling. The ability to solve the problem is very important owned by students because by accustoming to solve problems in mathematics, the students are expected to solve problems in everyday life. The troubleshooting indicators in this study, understanding the problem, planning the problem, completing it according to plan and checking back according to Polya stage (1973). Mathematical modeling is useful to simplify the problem to make it easier to understand so that the solution can be given is the best solution.

Problem solving abilities and mathematical modeling of students cannot be separated from the students' early mathematical abilities. Mathematical early ability is a capability that students have based on the understanding of previous mathematical material. Each individual has different mathematical initial abilities even though in teacher's learning never differentiate in giving mathematical material. According to the researchers this is influenced by the readiness of students in learning, repetition of students in doing mathematics problems and feedback given 
students to math mathematics. Based on the explanation, this research would like to describe the ability of algebraic thinking on student problem solving indicators based on the students' early mathematical ability. The advantage is that teachers better understand students' difficulties in algebraic materials and can provide the best solution of the difficulties faced by students both in the form of learning methods and variations of the matter.

\section{Methodology}

This research is a qualitative descriptive research because it aims to describe the profile of students' algebraic thinking ability based on early mathematical ability. The preparatory stage in this research is to compile, the problem of mathematical preliminary skills which is a matter of prerequisite for the material of building flat side space, composing instrument of thinking algebra consisting of mathematical modeling problem, mathematical problem solving and generalization. Subjects in this study were students who had high, medium and low mathematical preliminary skills. Instrument in this research is a matter of algebraic thinking ability and interview guide which deepens student's answer. The data analysis procedure consists of data analysis (reducing data, exposing data and drawing conclusions) and describing the results of data analysis. Data analyses based on sub indicators of algebraic thinking ability are problem solving, mathematics modeling and generalization. This article will present algebraic thinking skills with indicators of problem solving skills (understanding problems, planning solutions, solving problems and reexamining) and mathematical modeling (creating a mathematical model of the problem).

\section{Findings}

\section{Problem}

"A cuboid has a length of $1 \mathrm{~cm}$ more than the width and height $1 \mathrm{~cm}$ shorter than the width. The surface area of the cuboid is $148 \mathrm{~cm}^{2}$. a. Write the equation of the surface area of the cuboid!, b. Calculate the cuboid volume!"

\section{Subjects With High MPS (ST)}

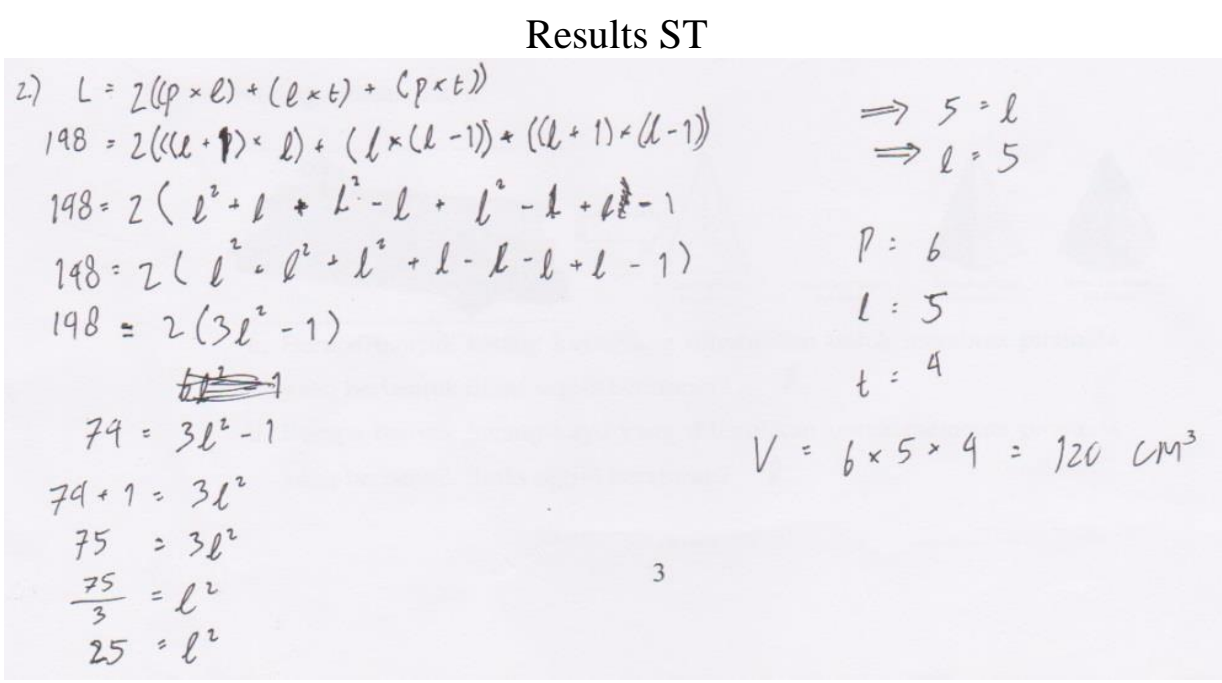


ST steps in solving the problem based on test results and interview results are as follows:

\section{Understand the Problem and Mathematics Modeling}

ST understands the problem by reading the problem slowly as it whispers its purpose to understand the given problem. The process of understanding the problem by writing down the information that is known and asked questions. ST can explain well what is known and asked. ST can model information correctly.

\section{Devise A Plan and Carry Out the Plan}

ST plotted the problem of modeling known information in algebraic form. After that calculate the width because that is known is the surface area of the cuboid. After obtaining the width it will be obtained high and long, so it can be calculated the volume of the cuboid. ST completed in accordance with the plan that has been told. ST is fast and meticulous in counting.

\section{Looking Back}

ST checks the settlement by recalculating rapidly and is sure that the ST answer is correct because when the length, width and height values are substituted into the volume formula and the surface area of the cuboid it will get the correct result.

\section{Subjects with Medium MPS (SD)}

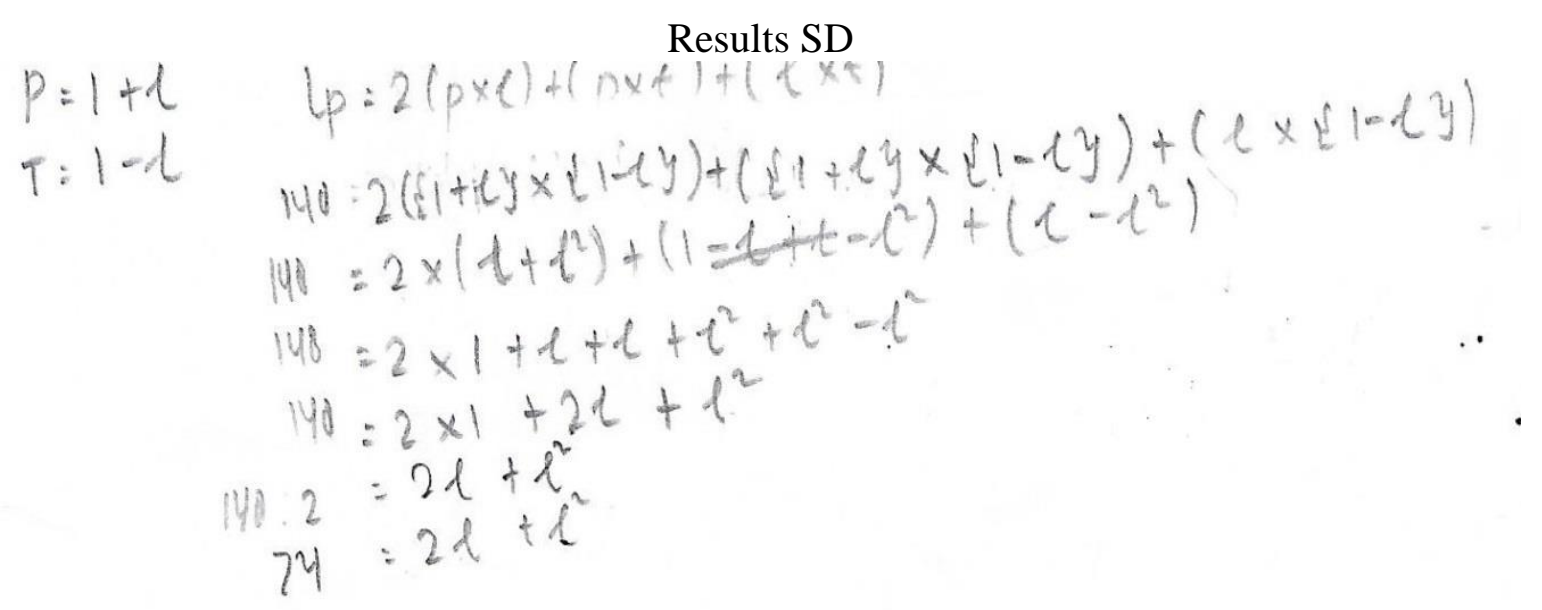

SD steps in solving problems based on test results and interview results are as follows:

\section{Understand the Problem and Mathematics Modeling}

SD understands the problem by reading the problem with a rather hard goal to understand the given problem. Habit elementary when faced with the story then read with a rather loud voice. The process of understanding the problem by writing down the information that is known and asked questions. On informas (height $1 \mathrm{~cm}$ shorter than width) SD model with $\mathrm{t}=1-1$. The 
researcher asked the elementary school whether confident with the modeling done, SD said that sure the information that SD understand in accordance with the given problem.

\section{Devise A Plan and Carry Out the Plan}

SD plotted the problem of modeling known information in algebraic form. SD understands that SD will get the width, length and height of the known surface area of the problem, but SD confuses in calculating the value (1) because in modeling the information is less precise. SD cannot solve the problem properly and appropriately.

\section{Looking Back}

SD did not solve the problem well so did not get the answer. So SD does not do the checking process of completion.

\section{Subjects with Low MPS (SR)}

The result of the SR response is not available because SR does not answer the given problem. SR steps in solving problems based on test results and interview results are as follows:

\section{Understand the Problem and Mathematics Modeling}

SR cannot understand the problem well, SR only reads the problem but does not explain the information that is known and asked in the matter. SR has no step in planning the settlement because SR is unaware of the cuboid surface area formula and cannot model the problem. This impacts SR cannot solve the problem well, so the process of re-examining the answer is not done by SR.

Translation of the difference of students' algebraic thinking ability which is classified as high early, medium and low mathematical ability can be poured in table form as follows:

Table Ability of Algebraic Thinking

\begin{tabular}{|l|l|l|l|}
\hline Algebraic Thinking Ability & ST & SD & SR \\
\hline Problem Solving & & \multicolumn{2}{l|}{} \\
\hline Understand the problem & $\sqrt{ }$ & $\sqrt{ }$ & $\sqrt{ }$ \\
\hline Devise a plan & $\sqrt{ }$ & $\sqrt{ }$ & - \\
\hline Carry out the plan & $\sqrt{ }$ & $\sqrt{ }$ & - \\
\hline Looking back & $\sqrt{ }$ & - & - \\
\hline Mathematical Modelling & & & \\
\hline Make the mathematical modelling & $\sqrt{ }$ & - & - \\
\hline
\end{tabular}


Based on the above table it can be seen that mathematical preliminary skills to give contribution to students in solving algebra thinking problem that is problem solving and mathematical modeling. Students who do not have a good mathematical foundation such as understanding mathematical formulas, doing algebraic processes and calculating well it will be difficult in understanding the next material. So this gives the impression that mathematics is a difficult subject.

\section{Discussions}

The students' mathematical preliminary skills affect students' ability to solve algebraic thinking problems. The results of this study indicate that students who have good mathematical preliminary skills, the student can understand the problem, model mathematics and solve problems well and have no barriers at all in the process of completion. This is because ST has basic mathematical concepts that can be applied in mathematical solution. While SD has a basic ability that is less good to the concept of mathematics. This is based on the results of the interview because it forgot the completion of the process of SD can not solve the problem well and precisely. The process of mathematical modeling of SD is less precise so that SD stop the settlement step due to confused with the process of calculating algebra. The completion process undertaken by SR is only in the early stages of trying to understand the given problem but for the mathematical modeling step, planning and resolving the SR problem has no way to resolve.

The low ability of concept comprehension and the low desire of students to practice solving mathematical problems make mathematics one of the most difficult and disliked lessons by students. Matter in mathematics is always related so that students do not understand the basic concept hence for concepts that will further trouble. So the teacher plays an active role to overcome the problem by always providing materials faster to be understood by students. In addition teachers can also provide practice from an easy problem then leads to a critical and creative problem so that students will be accustomed in practicing solving math problems.

\section{Conclusions}

The conclusion in this study is that students who have high mathematical preliminary skills(ST) able to understand, plan and complete according to plan well, ST check the answer by recalculating quickly and sure that the answer ST. Students who have mathematical preliminary skills(SD) are able to understand and plan the problem well, but in mathematical modeling, SD is less careful in understand one of the information provided so that in the process of solving the problem of SD experience confusion. Students who have low mathematical preliminary skills (SR) are only at the reading stage and do not understand so that the problem solving and mathematical modeling steps are not done well. This research is useful for teachers as facilitators in the classroom so as to facilitate learning that can enable students to remember the prerequisite material before the learning process of the material to be taught.

\section{Acknowledgements}

This research was carried out in Tangerang city junior high school with the guidance by the supervisor of Indonesia University of education, therefore, researchers say thanks to the Principal city of Tangerang and Indonesia University of education 


\section{References}

[1] Kieran, C. (2004). Algebraic Thinking in the Early Grade: What Is It? The Mathematics Educators 2004, Vol 8 No 1.139-151.

[2] Lew, H.C (2004). Developing algebraic thinking in early grade: case study in korean school mathematics. The Mathematic Educator, 8; 1(2004).

[3] Polya, G. (1973). How to Solve It a New Aspect of Mathematical Method. New Jersey: Princeton University Press.

[4] Radford, L. (2006). Algebraic Thinking and Generalization of Pattern: t a Semiotic Prespective. Proceedings of Twenty Eigh Annual Meeting of the North American Chapter of the International group for the Psychology of Mathematics Education.

[5] Radford, L. (2012). Early Algebraic Thinking Epistemological, Semiotic and Developmental Issues. 12th International Congress on Mathematical Education. 815 July, 2012, COEX, Seoul, Korea.

[6] Wilujeng, Hestu. (2016). Algebra Readiness Analysis of Junior High School Students Class 7. UNISSULA PRESS.

*Corresponding author.

E-mail address: hestuwilujeng@ gmail.com 